\title{
Fragmentation of displacement cascades into subcascades: a molecular dynamics study
}

by E. Antoshchenkova ${ }^{1,}{ }^{*}$, L. Luneville ${ }^{2,3}$, D. Simeone ${ }^{1,3}$, R. E. Stoller ${ }^{4}$, M. Hayoun ${ }^{5}$

${ }^{1}$ CEA, DEN, SRMA, LA2M, Matériaux Fonctionnels pour l'Energie, CNRS-CEA-ECP, CEN Saclay, F-91191 Gif sur Yvette, France

${ }^{2}$ CEA, DEN, SERMA, LLPR, Matériaux Fonctionnels pour l'Energie, CNRS-CEA-ECP, CEN Saclay, F-91191 Gif sur Yvette, France

${ }^{3}$ CNRS-CEA-ECP, Laboratoire SPMS, Matériaux Fonctionnels pour l'Energie, Ecole Centrale de Paris, F-92292 Châtenay Malabry, France

${ }^{4}$ Materials Science and Technology Division, Oak Ridge National Laboratory, Oak Ridge, TN 37831-6114, USA

${ }^{5}$ Laboratoire des Solides Irradiés, École Polytechnique, CEA-DSM, CNRS, F-91128

Palaiseau, France

${ }^{*}$ Corresponding author. Tel.: +33169334471

E-mail address: ekaterina.antoshchenkova@polytechnique.edu

\begin{abstract}
The fragmentation of displacement cascades into subcascades in copper and iron has been investigated through the molecular dynamics technique. A two-point density correlation function has been used to analyze the cascades as a function of the primary knock-on (PKA) energy. This approach is used as a tool for detecting subcascade formation. The fragmentation can already be identified at the end of the ballistic phase. Its resulting evolution in the peak damage state discriminates between unconnected and connected subcascades. The damage zone at the end of the ballistic phase is the precursor of the extended regions that contain the surviving defects. A fractal analysis of the cascade exhibits a dependence on both the stage of the cascade development and the PKA energy. This type of analysis enables the minimum and maximum displacement spike energies together with the subcascade formation threshold energy to be determined.
\end{abstract}




\section{Introduction}

Initially, the interest in displacement cascades was motivated by the nuclear industry, but nowadays cascade damage production is also of significant interest to the electronics industry through the ion implantation technique [1,2]. The principal differences between the nuclear and ion beam applications reside in the energy and mass of the particle that initiates the cascade. It should be noted that ion beams can also be used as a tool to investigate the mechanisms of radiation damage induced by neutron irradiation [3].

The processes of radiation damage, from initial defect production to microstructure evolution, occur over a wide range of time and size scales. To understand the drastic changes in materials properties, the primary damage state is a crucial stage that can only be investigated through atomic scale simulation methods. Despite over half a century of simulations, radiation damage formation is not completely understood. The molecular dynamics (MD) technique provides the most realistic description of the primary damage state $[4,5]$. Primary damage is produced in displacement cascades by the recoil of primary knock-on atoms (PKAs). For PKA energies very much larger than $100 \mathrm{keV}$ [6], simulations based on the binary collision approximation (BCA) [7] provide a model of the collisional phase of a cascade. However, such an approximation produces only a rough description of the defects; the thermal spike is missing [4].

Depending on the PKA energy, a displacement cascade can divide or not into several damage zones called subcascades [8,9]. This fragmentation was demonstrated both experimentally [10-12] and by BCA simulation [13-16]. Subcascades have been reproduced $[17,18]$ or predicted $[19,20]$ in MD simulations and appear regularly in recent articles with increasing damage energy $[3,6,21,22]$. Although many researchers have focused on the role of SIA (self-interstitial atom) clusters [23], the subject of subcascade formation has not really been well investigated. Typically, some kind of qualitative analysis would note that high-energy cascades consist of a few subcascades, but such analysis was purely descriptive and based on simple visualization of the cascades. Therefore, since the number of subcascades formed by a PKA may be an important factor in determining the damage structure, there is interest in determining how the phenomenon of point defect clustering may be influenced by subcascade formation.

Furthermore, one practical implication of subcascade formation is that very high-energy cascades break up into what looks like a group of lower energy cascades [3, 24].

This observation has led to the practice of simulating very high energy cascades as multiple cascades of lower energy [24].

In this work, we have performed numerous MD simulations in copper and iron, increasing the PKA energy until subcascade formation was observed as expected from previous BCA calculations $[15,16]$. Indeed, the idea is to focus on energies at which the cascade fragmentation begins. We found a physical quantity able to detect the existence of subcascades: a two-point density correlation function. A temporal cascade evolution and a relation between the defect cluster formation and subcascade formation will be also discussed. 


\section{Methods}

\section{II.1 MD computations}

The modeling of the copper interactions relies on an empirical n-body potential [25] of the tight-binding type $[26,27]$ that satisfactorily reproduces its physical properties. The MD computations have been performed in the microcanonical ensemble. The simulated system is a cubic box of size $L \Varangle \chi$ with $\{100\}$ faces, where $L \backslash X L=1$ is a unit cell of 4 atoms. When increasing the PKA energy from $0.2 \mathrm{keV}$ to $20 \mathrm{keV}$ we used three different sizes for the simulation box $(L=22, L=40, L=63)$ containing 42592, 256000 or 1000188 copper atoms, respectively. The MD time step was scaled to the fastest atom in the simulation system and varies by two orders of magnitude over the evolution of the cascade. Periodic boundary conditions were employed, and a Langevin thermostat [28] was applied by rescaling the velocities of atoms in the four outermost planes of the sides of the box. Energy leaving the cell was thus prevented from re-entering at the opposite boundary. The width of the boundary layers was tested and determined to be sufficient to prevent the cascade from self-interacting.

Initially, equilibrium configurations of the copper crystal for every box size have been generated by MD runs of $10 \mathrm{ps}$ at a temperature of $300 \mathrm{~K}$ and a pressure close to zero for a lattice parameter, $a$, of $0.36358 \mathrm{~nm}$. These equilibrated configurations were then used as the starting points for cascade simulations. Statistical variability was introduced by further evolution of the equilibrium prior to the initiation of a cascade.

The displacement cascades were initiated by transfer of kinetic energy to a single copper atom either at the center or near a side of the cell, depending on simulation cell and PKA energy. This simulates the desired recoil. The simulation box was sufficiently large for the cascade to be contained within the cell. In all simulation runs, the momentum transfer has always been aligned along the $<001>$ or $<111>$ directions of the FCC lattice. In this kind of simulation $[3,29,30]$, the PKAs are generally introduced along high-index crystallographic directions such as $\langle 123\rangle,<135\rangle$ or $<345>$. Our unusual choice of PKAs directions favors the channeling which occurs in preference along these crystallographic directions. Not avoiding the channeling increases the probability of finding subcascades [13], but also the number of Frenkel defects [31].

The evolution of the damage cascades were examined for about 15-18 picoseconds following the primary knock-on event. Furthermore, a few high-energy cascades were continued until 100 ps. During this stage, the positions of all atoms were recorded periodically.

It is known [32] that a cascade develops in three distinct stages. The first one is a ballistic phase, which involves high energy binary collisions and lasts about a few tenths of picoseconds. The second is a thermal spike, which is characterized by short range movements of a large number of atoms within a roughly spherical volume and lasts 13 ps. At this stage, the cascade reaches its peak damage state with the maximum cascade size and number of defects. Then, the heat is dissipated and the affected zone recrystallizes. This is the relaxation phase of the cascade, which can last few picoseconds. At the end of this phase, the majority of residual or surviving defects are found in defect clusters. To follow the evolution of lattice damage we will focalize on the basic states of 
the cascade development: end of the ballistic phase, the peak damage state and the MD final state of damage.

The details on MD computations of iron are discussed elsewhere $[3,30]$.

\section{II.2 Defect analysis}

Some procedure must be applied to determine which of the atoms should be defined as being in a defect state for the purpose of visualization and analysis. The identification of point defects in our simulations is mainly based on a simple geometric criterion. A sphere with a well-suited radius is centered on each perfect lattice site. When the spherical volume is empty, there is a vacancy on the site. If an atom does not belong to any atomic site, it is an interstitial atom.

To count the surviving defects in the final state of damage, the radius value is chosen in such a way that an atom which has left a regular site is discriminated from an atom with a large magnitude vibration at the equilibrium temperature of $300 \mathrm{~K}$. In this case, the value of the radius of the sphere was at least 0.15 times the lattice parameter $(a)$. For the counting of point defects at the end of the ballistic phase, the radius value must be increased since the system heats up. A suitable value for the radius is $0.25 a$.

For the analysis of the system at the peak damage state, we found that using only the simple geometric criterion is not sufficient for PKA energies above $2.5 \mathrm{keV}$. Indeed, in this state the cascade core is liquid-like while the neighboring lattice undergoes a phase of compression and expansion due to a moving pressure wave [33]. A temperature criterion was thus added in order to discriminate between the displacements of hot interstitial atoms from displacements of cold atoms due to this phenomenon. If an atom is farther than $0.25 a$ from the closest crystalline site and has a kinetic energy less than $0.08 \mathrm{eV}$, it is identified as occupying the site and no defect is counted.

The number of Frenkel defects obtained for different PKA energies is shown in Fig. 1. Their values are in agreement with previous work [19, 20, 34, 35].

\section{II.3 Two-point density correlation function}

After looking for a physical quantity able to detect the cascade fragmentation, we arrived at a two-point density correlation function. This function was taken from studies of the diffusion limited aggregation process [36] and also was calculated for high energy displacement cascades obtained within the binary collision approximation $[15,16]$ :

$$
C(\mathbf{r})=\frac{1}{N}\left\langle\sum_{\mathbf{y}} \rho(\mathbf{y}) \rho(\mathbf{r}+\mathbf{y})\right\rangle_{\Omega(\mathbf{r})}
$$

where $\mathrm{N}$ is the number of collisions in the displacement cascade, $\rho(\mathbf{y})$ is equal to 1 only

if there is a collision at a distance $\mathbf{r}$ and zero otherwise, and \langle\rangle$_{\Omega(\mathbf{r})}$ denotes the average over all solid angles $\Omega(\mathbf{r})$ generated by all collisions occurring in a displacement cascade. 
$C(\mathbf{r})$ only depends on the distance between two different collision points, $r=\left|\mathbf{r}_{i}-\mathbf{r}_{j}\right|$, and then reduces to $C(r)$. Moreover, the function follows a simple scaling law, where $C(r) \sim r^{-\beta}$. From the exponent $\beta$ of this correlation function a fractal dimension $D$ can be calculated, since $D=-\beta+d$, where $d$ is the Euclidean dimension of the simulated system. In the analysis of the MD trajectories, the vacancies are used to identify where the collisions occurred.

\section{Results and discussion}

\section{III.1. Analyzing multiple cascades}

Our idea is to compare the results of a few simultaneous cascades whose individual energies sum to a given value with a single cascade with the same total energy. The simultaneous cascades are initiated exactly at the same time and in the same simulation box. The ultimate purpose is to build an analysis tool which will be used as a reference in the detection of subcascades of $\S$ III.2.

We performed a series of MD simulations in copper in which the total PKA energy is $5 \mathrm{keV}$ : a single cascade of $5 \mathrm{keV}$, two cascades of $2.5 \mathrm{keV}$, and three cascades of $1.67 \mathrm{keV}$ in the simulation box. The choice of $5 \mathrm{keV}$ is motivated by the fact that such an energy: i) is below the subcascade threshold energy, $E_{c}$, [15] meaning free of subcascades and ii) produces a sufficient number of defects for statistical reasons.

The MD trajectories have been analyzed in the peak damage state via the two-point density correlation function ( $§$ II.3). The distance, $r$, is normalized by the largest dimension of the damaged region, $R_{\max }$, which corresponds to the largest distance between two vacancies.

Figure 2 displays the differences between a single, two or three cascades with the same total energy of $5 \mathrm{keV}$. The single case provides the characteristic shape of $C(r)$ for a non fragmented cascade in the regime of maximum defect-density. In such a regime, each cascade atom is displaced from its lattice site [8]. The curve for the two unconnected cascades (of the same size) displays a deep well absent in the single cascade curve. It is located at a distance corresponding to the cascade diameter, $R$. In this region, the distances $r$ are mainly sampled within the two cascades (intra) but also include some distances less than $R$ between the two cascades. A pronounced peak is located at the distance $\Delta$ which is the distance separating the two cascade centers. The well and the peak are separated by the distance $\delta$ matching the shortest distance between the edges of the two cascades. Therefore, the two cascades can be detected in $C(r)$. For the same cascade size $R$, we observed that as the distance $\delta$ increases the well get deeper. In the case of three cascades, we observe two wells in $C(r)$. For two cascades of different sizes (not displayed), a deep well is found at a distance corresponding to the diameter of the largest cascade.

The results obtained clearly show that the two-point density correlation function allows identifying the presence of several cascades and to estimate their characteristic sizes. This demonstrates that $C(r)$ can be used as a tool to detect the signature of subcascade formation. 
III.2. Cascade fragmentation at the peak damage state

At the peak damage state we observe the maximum cascade size and number of defects. This state is well suited to analyze the presence of possible cascade fragmentation. We will emphasize that the segments of fragmented cascades can be connected or unconnected.

We carried out 25 runs of MD simulations with PKA energies of 10 and $20 \mathrm{keV}$ providing the opportunity for subcascade formation. At the peak damage state, the cascades can be classified in three ways determined by the shape of their $C(r)$. Examples of such different behaviors, obtained from individual trajectories, are shown in Fig. 3. The first example, referred to hereafter as unconnected type behavior, displays a deep well revealing the clear fragmentation of the cascade into two unconnected subcascades. The separation distance between them can be estimated from the positions of the well and the peak. The value for this cascade is around $4 \mathrm{~nm}$. Fig. 4(a) gives the corresponding structure of the cascade from the location of the defects. We observe two separate damaged zones in full agreement with the $C(r)$ analysis. In the second example of $C(r)$ of Fig. 3, the unfragmented type, there is no fragmentation since the curve behaves similar to the single cascade of Fig. 2. The associated structure of the cascade is given in Fig. 4(b) and does not display any subcascade. The $C(r)$ of the connected type of Fig. 3 is intermediate between unconnected and unfragmented types. Its curvature is different from that of the unfragmented type. It follows the curve of the unconnected type at short distances $(r /$ $R_{\max }<0.3$ ) but does not exhibit a well. This corresponds to a fragmentation in connected subcascades sometimes called "lobes". This last term originated from a study by Heinisch [13].

For a given PKA energy, when $C(r)$ is averaged over numerous MD configurations, its behavior is more difficult to interpret. Indeed, the behavior is weighted by the contributions of the three types: unconnected, unfragmented and connected. The weight of each contribution depends on the cascade energy. At $10 \mathrm{keV}$, the cascades are not all fragmented and the unconnected type is uncommon whereas for $20 \mathrm{keV}$ all cascades are fragmented.

Averaged $C(r)$ for PKA energies ranging from 0.2 to $20 \mathrm{keV}$ are shown in Fig. 5. With increasing cascade energy in the range $0.2-5 \mathrm{keV}$, the value of $C(r)$, increases as shown by Fig. 5a. The trend is inversed for increasing energies from $5 \mathrm{keV}$ (see Fig.5b). We thus find the influence of the cascade energy on the density of Frenkel defects of which the maximum, also called displacement spike, is reached at about $5 \mathrm{keV}$. Since the $C(r)$ for 2.5, 5 and $10 \mathrm{keV}$ are very close together, the corresponding defect density reaches a plateau value between minimum spike energy of $2.5 \mathrm{keV}$ and a maximum spike energy of $10 \mathrm{keV}$. For such a maximum, the displacement cascade affects all the atoms initially located in the damage region. The behavior change occurring in the maximum zone suggests also a transition between regimes with or without cascade fragmentation. This suggests a threshold energy, $E_{c}$, greater or equal to the spike energy maximum. Heinisch and Singh [5] concluded similarly within the BCA approximation. They found that the existence of subcascades above the threshold energy, can be empirically verified from the vacancy distributions. In their work on fractal geometry of collision cascades, Rossi 
et al. [37] even claim that the maximum spike energy equals the threshold, $E_{c}$, while the minimum spike energy is $E_{c} / 2$.

\section{III.3. MD Final state of damage}

We now consider the evolution of the cascade fragmentation in the final state of damage. We extend the MD simulations up to 15-18 ps. The number of Frenkel defects becomes stable even for the highest energy under study.

In the MD final state of damage, we observe either defect clusters of different sizes or isolated defects that can be gathered in separated regions. All these surviving defects are inherited from the cascade fragmentation occurring in the peak damage state. The results averaged over several configurations (at least 10 for $\mathrm{Cu}$, and at least 6 for $\mathrm{Fe}$ ) for copper and iron are displayed in Fig. 6 . The $C(r)$ of $20 \mathrm{keV}$ copper cascades in the peak damage state is used as a reference curve to analyze all other $C(r)$ in the final state of damage. The defect density obviously decreases after the thermal spike stage has ended and also with increasing PKA energy (all energies are not displayed). This is supported by a detailed analysis of the dependency on the PKA energy (20 keV-2 MeV) of the defect density in iron by Dierckx in a BCA study [14]. For copper and iron in the $20 \mathrm{keV}$ final state, the curves are similar at large distances while a significant difference is observed for short distances. The higher defect density at short distances for copper indicates that distances are sampled within defect clusters for which the size is larger in copper than in iron. This means that two different relaxation regimes occur in the two metals and is consistent with earlier observations of differences in clustering between iron and copper [20]. Examples of clusters obtained in copper and iron cascades in the final damage state are given in Fig. 7.

The iron $C(r)$ exhibits a shallow well suggesting the presence of two separate defect regions in Fig. 6. Since $C(r)$ is an average, this signifies that such defect regions correspond to the most likely configuration. This can be seen in the configuration of Fig. $7 \mathrm{~b}$. In the case of copper, the numerous clusters can be counted based on the wells of the $C(r)$ of individual MD trajectories (not displayed) but the subcascade fragmentation cannot be clearly established.

\section{III.4. Temporal evolution}

It is interesting to follow the temporal evolution of possible cascade fragmentation through the three stages discussed above: at the end of the ballistic phase (around $0.1 \mathrm{ps}$ ), in the peak damage state and in the MD final state of damage. It is important to mention that the thermal spike phase cannot be modeled within BCA simulations [5]. We now consider the links between these three important stages. Is it possible to predict the type of cascades from analyzing the ballistic phase? Are the surviving defects in the final state of damage useful to capture the cascade fragmentation at the preceding moment?

The three types of cascades previously defined in the peak damage state (§ III.2) are also investigated at shorter (end of the ballistic phase) and longer times (final state of damage). Their $C(r)$ are given in Fig. 8. At the end of the ballistic phase, the $C(r)$ of unconnected and connected subcascades are similar and differ clearly from the $C(r)$ of 
an unfragmented cascade. In the former, the slope is constant for values of $r / R_{\max }$ ranging between 0.1 and 0.5 whereas in the last one we observe a pronounced curvature characteristic of a single cascade. Therefore, at this stage, we can predict whether or not the fragmentation will occur, but we are not able to discriminate the evolution towards connected or unconnected subcascades. This point is corroborated by the snapshots in Fig. 9. Indeed, the pictures at the end of the ballistic stage exhibit a precursor aspect of the evolution to the peak damage state. Especially, the shape of the damage zone at the end of the ballistic stage is encompassed and enlarged at the following state.

A representative example of subsequent evolution of unconnected subcascades in the final state of damage is seen in Fig. 8a and Fig. 9a. The two subcascades evolve in two separate damaged regions clearly identified in both the $C(r)$ and the associated snapshots. Otherwise, for all types of cascades, the number of surviving defect clusters can be counted from the number of successive wells and peaks of $C(r)$.

These defect clusters are located solely in the damage region defined by the ballistic phase and the larger ones are preferentially created at the end of the ballistic path. Another place for large clusters is the overlap of lobes of connected subcascades. This description of the damage evolution is compatible with the one of Calder et al. [33].

The size of the cascade can be characterized by the largest dimension of the damaged region, $R_{\max }$. Its mean value as a function of the PKA energy at different stages of the cascade development is plotted in Fig. 10. In the final state of damage, the behavior is linear on a log-log scale with slope values that are similar for copper and iron. For a PKA energy of $5 \mathrm{keV}$, a change of slope is observed in the case of copper, at the end of the ballistic phase and in the peak damage state. This corresponds in the peak damage state to the achievement of the plateau value in the displacement spike and corroborates the transition between regimes with or without cascade fragmentation already identified in Fig. 5. It is worth mentioning that: i) the behavior of the cascade size at the end of the ballistic stage is the precursor of its evolution in the peak damage state and ii) the cascade sizes are similar at the end of the ballistic stage and in the final state of damage.

An effective fractal dimension, $D$, of the cascade can be calculated from the initial slope of $C\left(r / R_{\max }\right)$ averaged over several configurations, $\beta=D-d=D-3$ where $d$ is the dimension of the space. $D$ obtained as a function of the PKA energy in three stages of the cascade development are displayed in Fig. 11. Our MD calculations show that the fractal dimension is energy dependent. $D$ also changes during the cascade evolution reaching the maximum value at the peak damage state and then decreasing. In both the early and peak stages of the cascade development, the fractal dimension reaches plateau values in the energy range of $2.5-10 \mathrm{keV}$. At $10 \mathrm{keV}$, the $D$ value is close to the space dimension (3) of the simulated system. This means that the displacement spike conditions are met as shown by Cheng et al. [38]. The minimum spike energy would then be defined as the lower boundary of the energy range of the $D$ plateau while the maximum spike energy would correspond to the threshold energy for cascade fragmentation as proposed by Heinisch et al. [5]. In the final state, the fractal dimension trend for copper is driven by a transition between two regimes clearly seen at 2.5-5 keV in Fig. 11. The first regime, at low energies, consists of small defect clusters and isolated defects whereas in the second one it appears that larger clusters account for most of the surviving defects. This 
transition occurs when the displacement spike is formed. The clustering fraction is estimated to be around $80 \%$ by many authors $[29,39]$. A different trend is found for iron whose clustering is less efficient. The minimum value of $D$ seen in Fig. 11 for an energy of $20 \mathrm{keV}$ can be compared to the MD estimate above $10-20 \mathrm{keV}$ of the thereshold energy of subcascade formation by Stoller et al. [3, 40].

\section{III.5. Discussion}

Subcascades are damaged regions separated by slightly damaged zones [see for example $5,14,17]$. This can be clearly seen in the time sequence of Fig. 3 of [40]. One concept of subcascade formation relies on the fact that a recoil is likely to produce several isolated thermal spikes in the form of small liquid-like pockets rather than a single dense one [41]. That means that the peak damage state is well suited to analyze the presence of subcascades. The concept is clear in the case of unconnected subcascades separated by center-to-center distances greater than their own size. Nevertheless and contrary to Sato et al., [12] we think that connected subcascades must be taken into account. Most of them do not look like a spherical unfragmented cascade. Their influence on the microstructure is really different from that of a single cascade. The connected subcascades lead to a specific space repartition of the surviving defects. Large surviving clusters often appear at the locations of the connections observed in the peak damage state. Such trapping zones for defects have also been evidenced by Calder et al. [33].

The subcascade formation threshold energy, $E_{c}$, can be estimated from the behavior change of $C(r)$. The lower energy for which the signature of the cascade fragmentation appears provides a first estimate. A more accurate value is obtained with the evolution of the fractal dimension as a function of energy in all stages of the cascade development (Fig. 11). In the peak damage state, a subcascade is created as early as the fractal dimension reaches the space dimension (3), that is to say when all the defects fill a given volume. The subcascade formation threshold energy is found to be $10 \mathrm{keV}$ for copper, in agreement with the value mentioned by Nordlund et al. [41]. For energies lower than this energy, no cascade fragmentation occurs. At the threshold energy, only a small fraction of the MD cascades are fragmented and most of them are connected. For energies greater than the threshold energy, all cascades are fragmented. Connected subcascades will always be present whatever the energy. This is corroborated by the MD simulations of the irradiation of airon crystals at PKA energies up to $500 \mathrm{keV}$ [21]. Our value of subcascade formation threshold energy for copper is in close agreement with the one calculated, as a function of the atomic number Z, by Monte Carlo in the BCA framework [15]. An alternative way to capture $E_{c}$ is to study the dependence of the number of surviving defects with the PKA energy. A change in the slope at 10-20 keV in iron is associated to a decomposition of the cascade into several subcascades [30]. For copper, such a behavior can only be guessed in Fig. 1. This confirms that the peak damage state is a better route to investigate the subcascade fragmentation.

The fractal approach applied to our MD data provides a fractal dimension $D$ that depends on the PKA energy and on the stage of the cascade development. For energies lower than the minimum spike energy, both $D$ and the cascade size increase. Between the minimum (2.5 keV) and maximum (10 keV) spike energies, both $D$ and the defect density reach plateau values in the peak damage state. Hence, each subcascade (connected or unconnected) corresponds to a displacement spike (one liquid-like 
pocket) and at high energies the whole cascade results in a multiple-spike formation. The properties of such a cascade are thus transmitted to the generated subcascades. In addition, the minimum and maximum spike energies lead to the minimum and maximum subcascade sizes, respectively. In the case of copper, we found and $12.1 \pm 0.7 \mathrm{~nm}$ for the limit sizes. That means the elementary bricks of the complete cascade are all known. Such a general description for high energies $(\geq 100 \mathrm{keV})$ implies that the cascade breaks up into multiple cascades of lower energy [3, 24, 37].

\section{Conclusion}

We have investigated the fragmentation of displacement cascades in copper and iron. The two-point density correlation function proves to be an interesting tool to analyze cascades as a function of the PKA energy. It detects the presence or absence of subcascades. At the end of the ballistic phase, the fragmentation can already be identified. Its subsequent evolution in the peak damage state allows discriminating between unconnected and connected subcascades. These latter ones cannot be ignored and must not be confused with unfragmented cascades.

The damage zone at the end of the ballistic phase is precursor of the extension of the regions containing the surviving defects. Additionally, the overlapping zones of connected subcascades, identified in the peak damage state, determine the emergence of large surviving defect clusters. Their number is not simply linked to the number of subcascades.

The fractal dimension $D$ of a MD cascade depends on the PKA energy and on the stage of the cascade development. Its evolution enables the determination of the minimum and maximum spike energies together with the subcascade formation threshold energy (estimated as $10 \mathrm{keV}$ for copper).

\section{Acknowledgements}

Support for Stoller at the Oak Ridge National Laboratory was provided by the Office of Fusion Energy Sciences, U.S. Department of Energy, under contract DE-AC05000R22725 with UT-Battelle, LLC. 


\section{References}

[1] W. Bolse, Mater. Sci. Eng. R. 12 (1994) 53.

[2] A. F. Tasch, Nucl. Inst. Meth. Phys. Res. 74B (1993) 3-6.

[3] Primary Radiation Damage Formation, R. E. Stoller, in Comprehensive Nuclear Materials, edited by R. J. M. Konings, vol. 1 (Elsevier, 2012) 293-332.

[4] K. Nordlund and F. Djurabekova, J. Comput. Electron 13 (2014) 122-141.

[5] H. L. Heinisch and B. N. Singh, Philosophical Magazine A 67 (1993) 407-424.

[6] K. Trachenko, E. Zarkadoula, I. T. Todorov, M. T. Dove, D. J. Dunstan and K. Nordlund, Nucl. Instrum. and Meth. B 277 (2012) 6-13.

[7] M. T. Robinson and I. M. Torrens, Phys. Rev. B 9 (1974) 5008.

[8] J. Brinkman, J. Appl. Phys. 25 (1954) 961.

[9] G. H. Kinchin and R. S. Pease, Rep. Prog. Phys. 18 (1955) 1-51.

[10] M. Kiritani, T.Yoshiie, S.Kojima and Y. Satoh, Radiation Effects and Defects in Solids 113 (1990) 75-96.

[11] Y. Satoh, M. Tsukada, H. Matsui and T. Yoshiie, J. Nucl. Mater. 329-333 (2004) 11851189.

[12] K. Sato, T. Yoshiie, Qiu Xu, Y. Satoh, E. Wakai, C. Kutsukake and T. Nishitani, Materials Transactions 46 (2005) 445-449.

[13] H. L. Heinisch, Radiation Effects and Defects in Solids 113 (1990) 53-73.

[14] R. Dierckx, J. Nucl. Mater. 144 (1987) 214.

[15] L. Luneville, D. Simeone and W. J. Weber, J. Nucl. Mater. 415 (2011) 55-60.

[16] D. Simeone, L. Luneville and Y. Serruys, Phys. Rev. E 82 (2010) 011122.

[17] T. Diaz de la Rubia and M. W. Guinan, Phys. Rev. Lett. 66 (1991) 2766.

[18] R. E. Stoller, G. R. Odette and and B. D. Wirth, J. Nucl. Mater. 251 (1997) 49-60.

[19] A.J.E. Foreman, W.J. Phythian, C.A. English, Phil. Mag. A66 (1992) 671.

[20] W.J. Phythian, R.E. Stoller, A.J.E. Foreman, A.F. Calder and D. J. Bacon, J. Nucl. Mater. 223 (1995) 245-261

[21] E. Zarkadoula, S. L. Daraszewicz, D. M. Duffy, M. A. Seaton, I. T. Todorov, K. Nordlund, M. T. Dove and K. Trachenko, J. Phys.: Condens. Matter 25 (2013) 125402.

[22] G. Martin, P. Garcia, L. Van Brutzel, B. Dorado and S. Maillard, Nucl. Instrum. and Meth. B 269 (2011) 1727. 
[23] B. N. Singh, M. Eldrup, A. Horsewell, P. Ehrhart and F. Dworschak, Phil Mag. A 80 (2000) 2629.

[24] A. Soudi, M. Hou, C. S. Becquart, L. Malerba, C. Domain and R. E. Stoller, J. Nucl. Mater. 419 (2011) 122-123.

[25] C. Rey-Losada, M. Hayoun and V. Pontikis, in Materials Theory and Modelling, edited by J. Broughton, P. Bristowe and J. Newsam, MRS Symposia Proceedings No. 291 (Materials Research Society, Pittsbourg, 1993) 549.

[26] G. J. Ackland and V. Vitek, in Atomistic Simulation of Materials: Beyound PairPotentials, edited by V. Vitek and D. J. Srolovitz (Plenum, New Yourk, 1989) 193.

[27] V. Rosato, M. Guillope and B. Legrand, Phil. Mag. A59 (1989) 321.

[28] N. Binggeli and J. R. Chelikowki, Phys. Rev. B 50 (1994) 11764.

[29] R. E. Voskoboinikov, Yu. N. Osetsky and D. J. Bacon, J. Nucl. Mater. 377 (2008) 385395.

[30] R. E. Stoller and A. F. Calder, J. Nucl. Mater. 283-287 (2000) 746-752.

[31] D. J. Bacon, A. F. Calder, J. M. Harder and S. J. Wooding, J. Nucl. Mater. 205 (1993) 52.

[32] L. Van Brutzel and J. P. Crocombette, Matter. Res. Soc. Symp. Proc. (2007) 981.

[33] A. F. Calder, D. J. Bacon, A. V. Barashev and Yu. N. Osetsky, Phil. Mag. 90 (2010) 863.

[34] M. Ojha, D. M. Nicholson, Kh. Odbadrakh, B. Radhakrishnan, R. E. Stoller and T. Egami, J. Phys.: Conf. Ser. 402 (2012) 012010.

[35] R. E. Voskoboinikov, Yu.N. Osetsky and D. J. Bacon, Nucl. Instrum. and Meth. B 242 (2006) 68-70.

[36] T. A. Witter Jr. and L. M. Sander, Phys. Rev. Lett. 47 (1981) 1400.

[37] R. Rossi, D. M. Parkin and M. Nastasi, J. Mater. Res. 4 (1989) 137.

[38] Y.-T. Cheng, M.-A. Nicolet and W. L. Johnson, Phys. Rev. Lett. 58 (1987) 2083.

[39] D. J. Bacon, F. Gao and Yu. N. Osetsky, J. Nucl. Matter. 276 (2000) 1.

[40] R. E. Stoller and L. R. Greenwood, J. Nucl. Mater. 271\&272 (1999) 57-62.

[41] K. Nordlund, M. Ghaly, R. S. Averback, M. Caturla, T. Diaz de la Rubia and J. Tarus, Phys. Rev. B 57 (1998) 7556. 


\section{Figure Captions}

Figure 1: Mean value of the number of defects (Frenkel pairs) as a function of the MD PKA energy. Peak damage state: copper, grey circles; copper from [19], black circles; iron from [34], open circles. Final state: copper, grey triangles; copper from [20, 35], black triangles; iron, open triangles. End of the ballistic stage: copper, grey squares.

Figure 2: two-point density correlation function, $C(r)$, for copper in the peak damage state, as a function of the distance, $r$, normalized by the largest dimension of the damaged region, $R_{\max }$. The total PKA energy of $5 \mathrm{keV}$ is used to generate one, two or three separated cascades.

Figure 3: two-point density correlation function, $C(r)$, for copper in the peak damage state, as a function of the distance, $r$, normalized by the largest dimension of the damaged region, $R_{\max }$. The PKA energies are $10 \mathrm{keV}$ : open squares or $20 \mathrm{keV}$ : open circles and crosses.

Figure 4: snapshots of three cascades represented by point defects in the peak damage state. (a) Unconnected type: subcascades, PKA energy of $20 \mathrm{keV}$; (b) unfragmented type: no subcascade, PKA energy of $10 \mathrm{keV}$; (c) connected type: subcascades, PKA energy of $20 \mathrm{keV}$. The $R_{\max }$ values are 19.7, 11.2 and $23.9 \mathrm{~nm}$, respectively.

Figure 5 : (Color online) two-point density correlation function, $C(r)$, for copper in the peak damage state, averaged over at least 10 MD configurations for each PKA energy. The arrows indicate the direction of increasing energies.

Figure 6: two-point density correlation function, $C(r)$, in the final state of damage , as a function of the distance, $r$, normalized by the largest dimension of the damaged region, $R_{\max } C(r)$ of copper at $20 \mathrm{keV}$ in the peak damage state is also given.

Figure 7: (Color online) snapshots of surviving defects in the final state of damage for a PKA energy of $20 \mathrm{keV}$. (a) copper, (b) iron. Both interstitials (large circles) and vacancies (small circles) are drawn.

Figure 8: Time evolution of the two-point density correlation function, $C(r)$, for copper as a function of the distance, $r$, normalized by the largest dimension of the damaged region, $R_{\max }$. Black line: end of the ballistic phase, open squares: peak damage state, open diamonds: final state of damage. (a) Unconnected type: subcascades, PKA energy of $20 \mathrm{keV}$; (b) unfragmented type: no subcascade, PKA energy of $10 \mathrm{keV}$; (c) connected type: subcascades, PKA energy of $20 \mathrm{keV}$.

Figure 9: (Color online) Superposition of snapshots of cascades represented by point defects for three stages of temporal evolution. Light gray (blue): end of the ballistic phase, black: peak damage state, gray (red): final state of damage. (a) Unconnected type: subcascades, PKA energy of $20 \mathrm{keV}$; (b) unfragmented type: no subcascade, PKA energy of $10 \mathrm{keV}$; (c) connected type: subcascades, PKA energy of $20 \mathrm{keV}$.

Figure 10: Mean value of the largest dimension of the damaged region, $R_{\max }$, as a function of the PKA energy. For copper, at the end of the ballistic phase: open squares, in the peak damage state: open circles, and in the MD final state of damage: full diamonds. For iron in the MD final state of damage: crosses. All lines are fittings of power functions. 
Figure 11: Fractal dimension , $D$, as a function of the PKA energy. For copper, at the end of the ballistic phase: open squares, in the peak damage state: open circles, and in the MD final state of damage: full diamonds. For iron in the MD final state of damage: crosses. All lines are guides for the eye. 


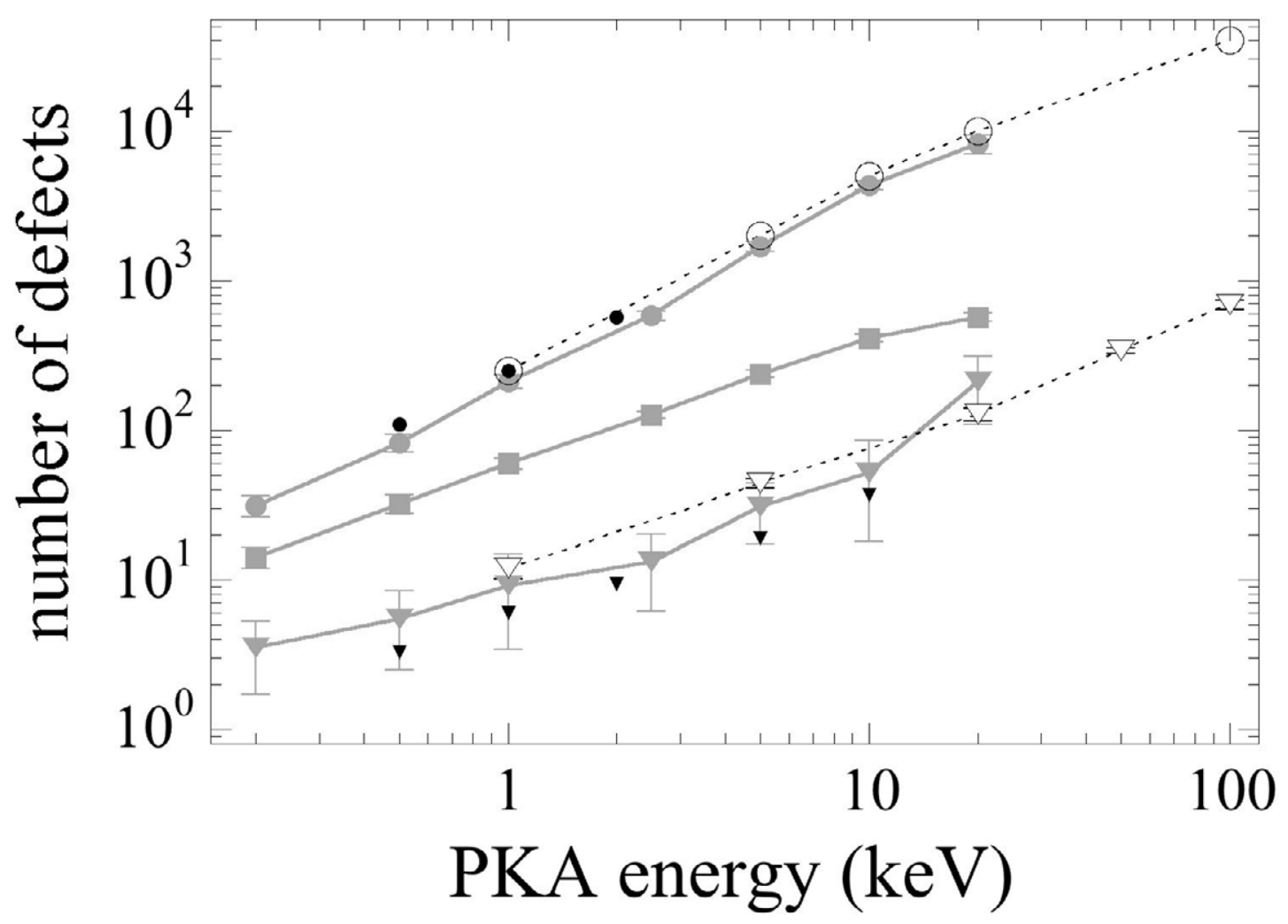

Figure 1 


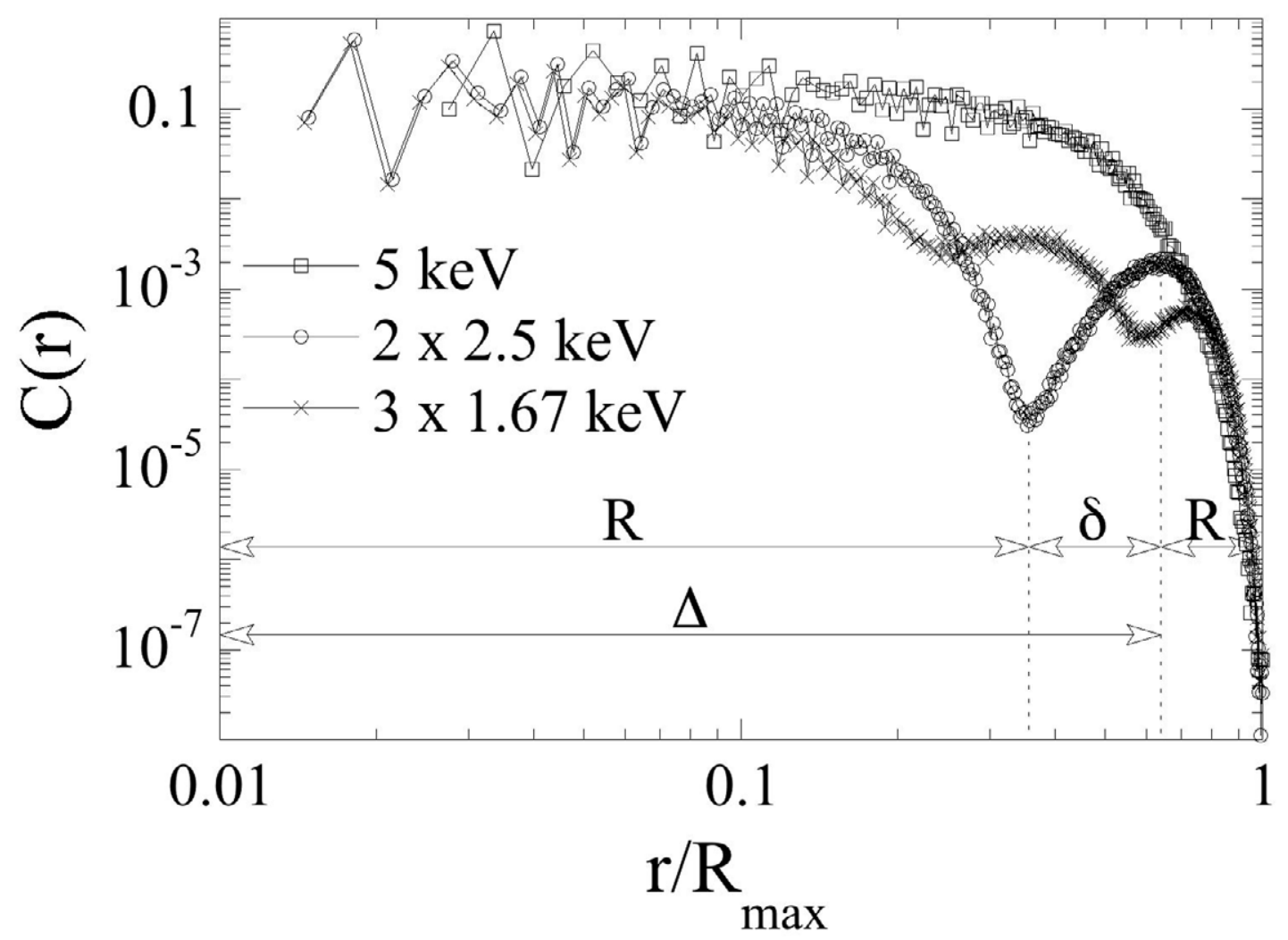

Figure 2 


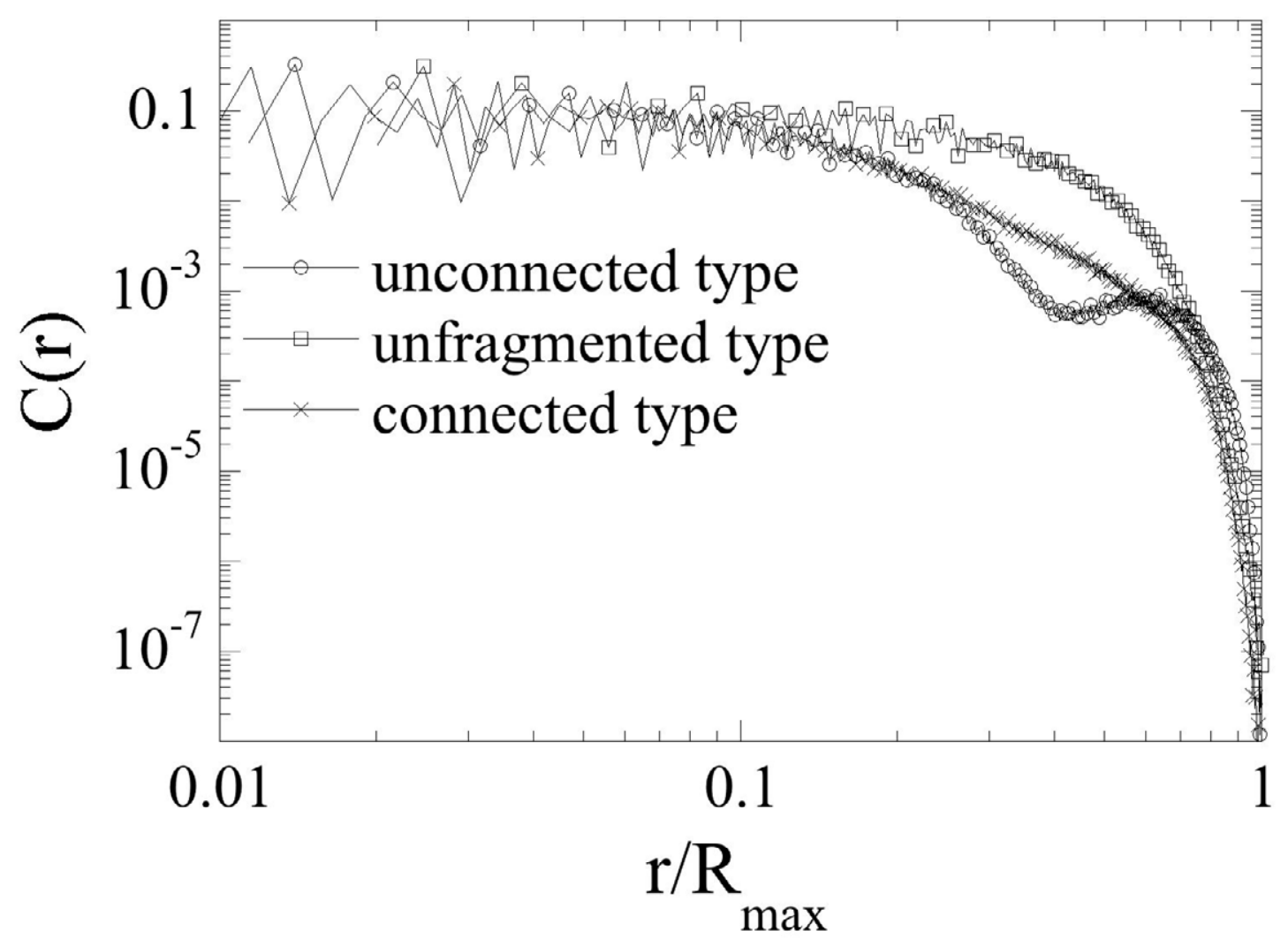

Figure 3 
(a)

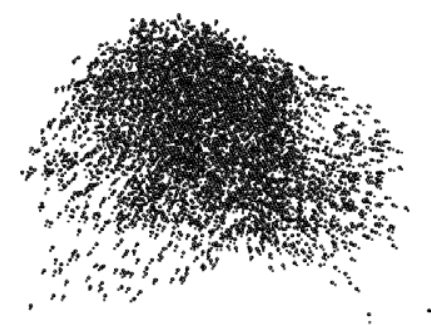

(b)
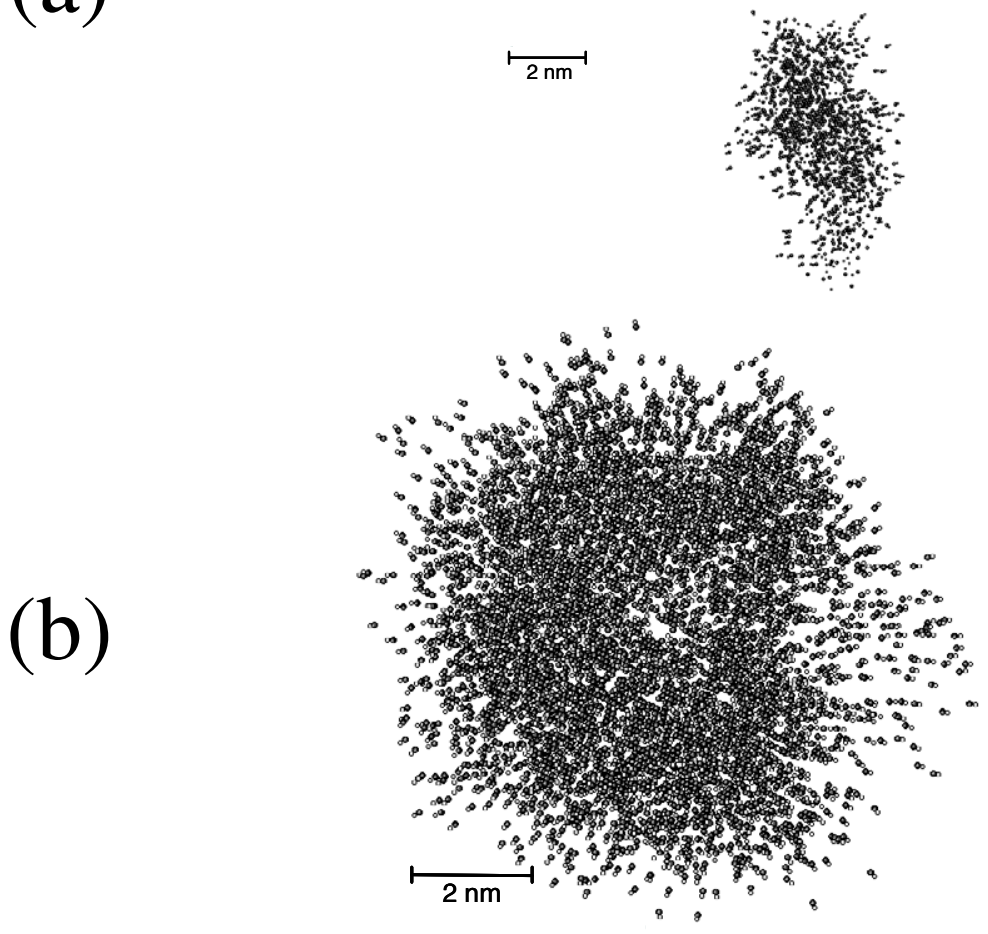

(c)

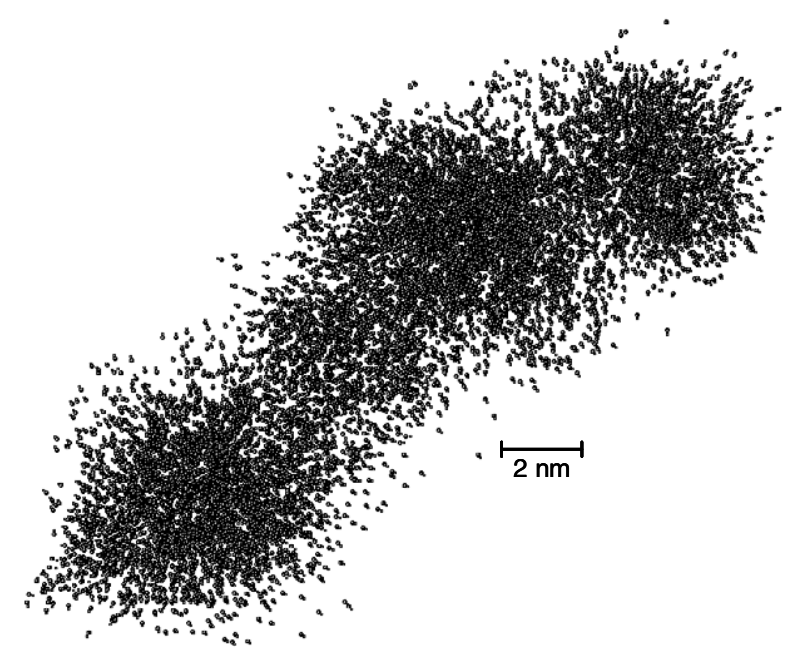

Figure 4 

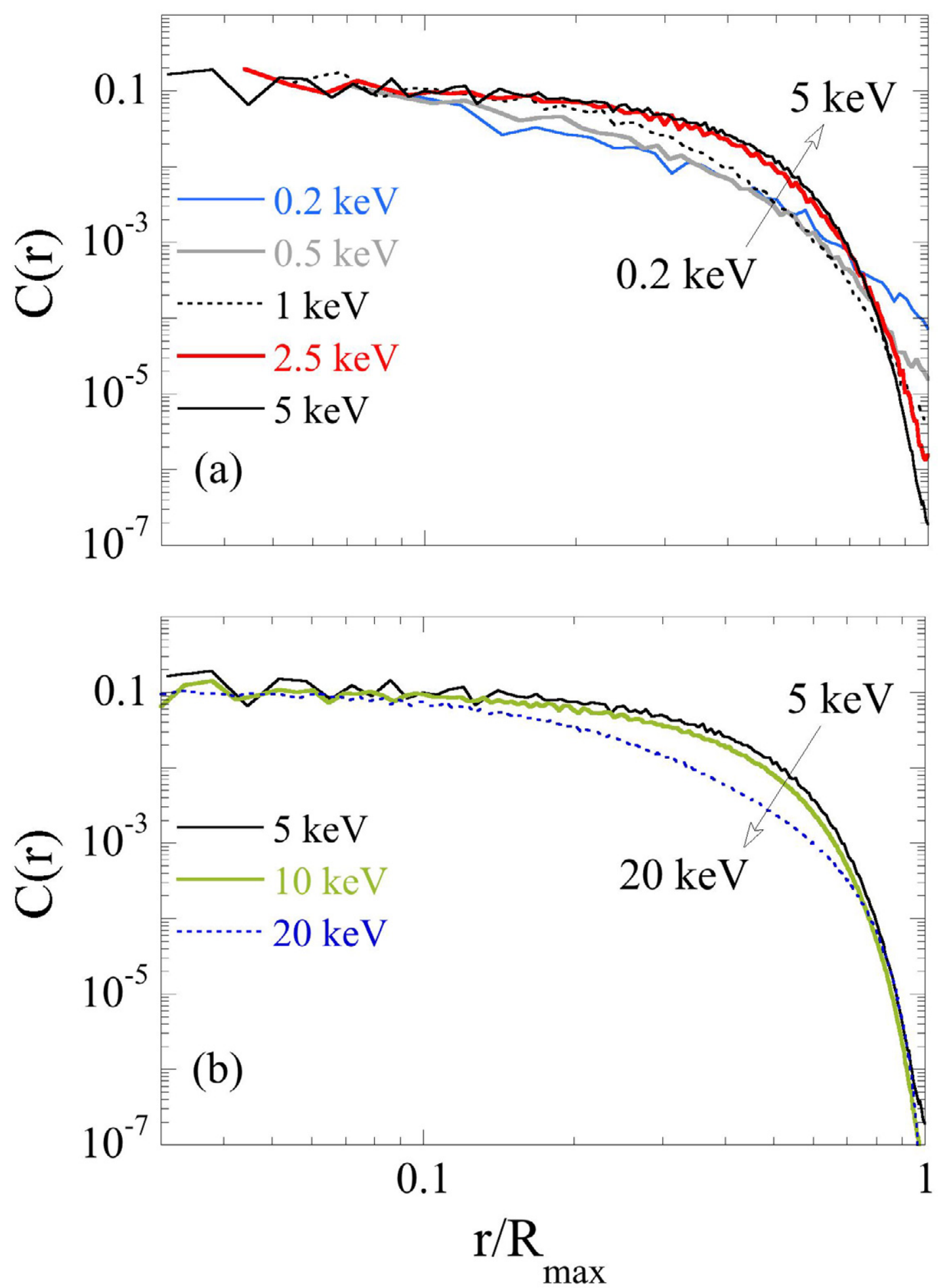

Figure 5 


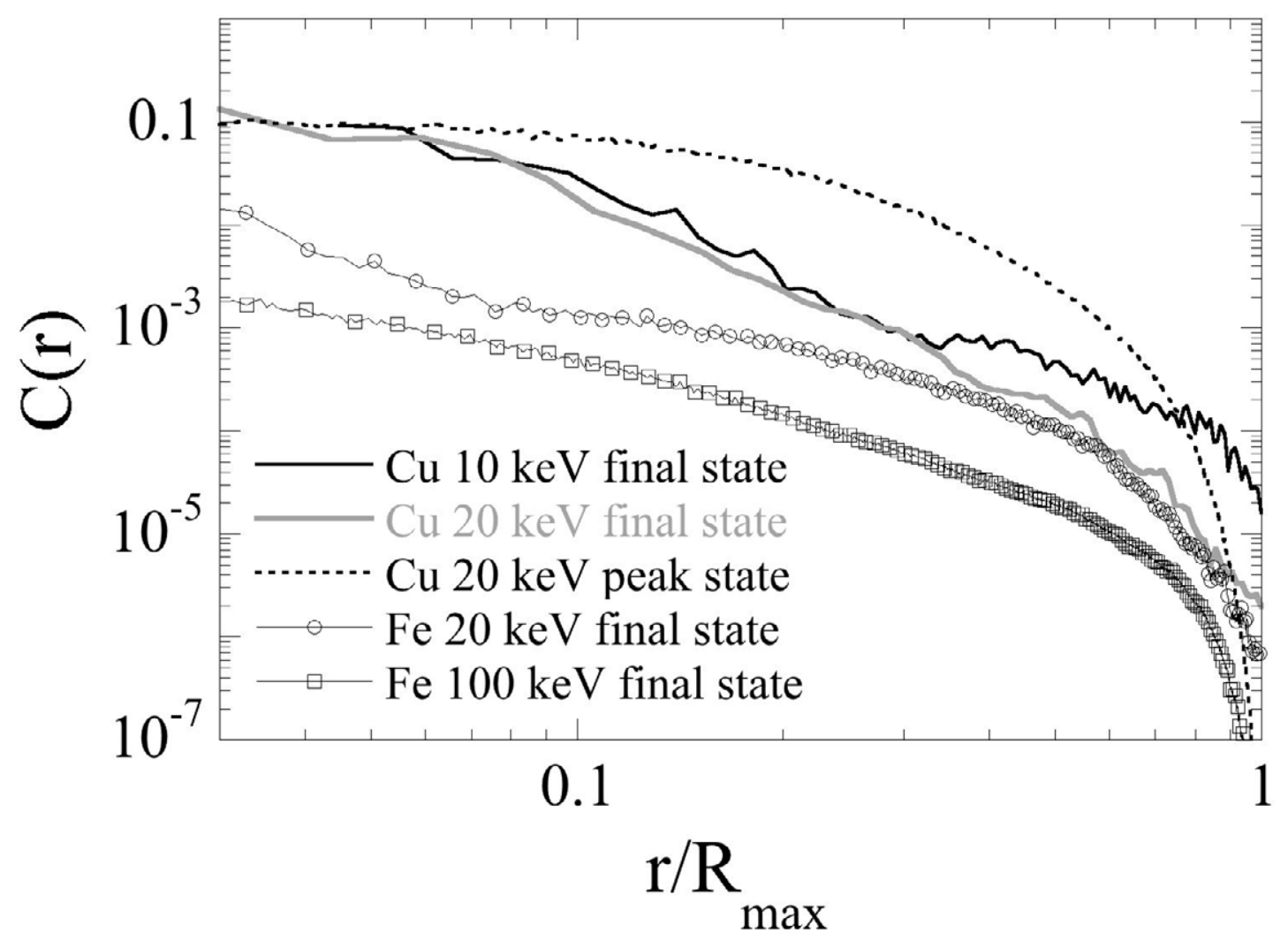

Figure 6 
(a)

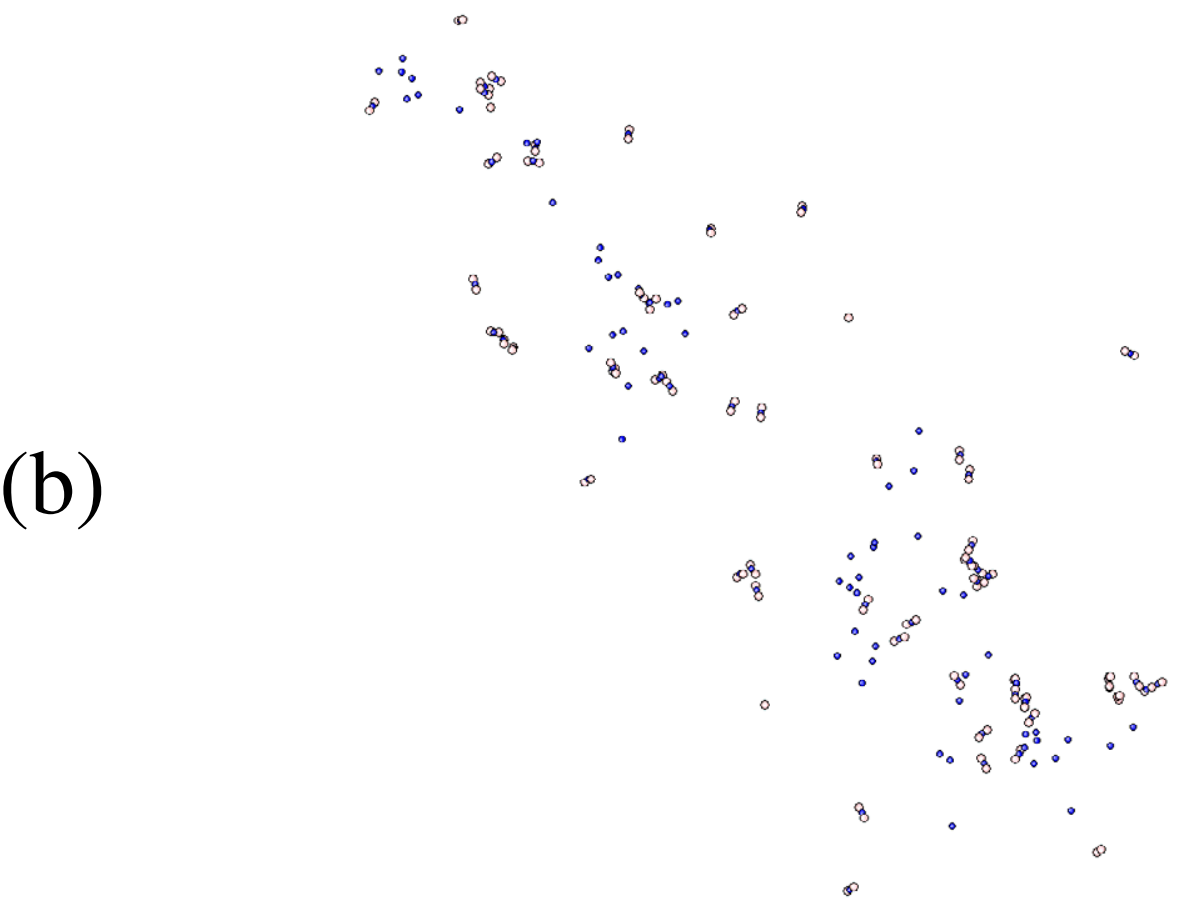

Figure 7 

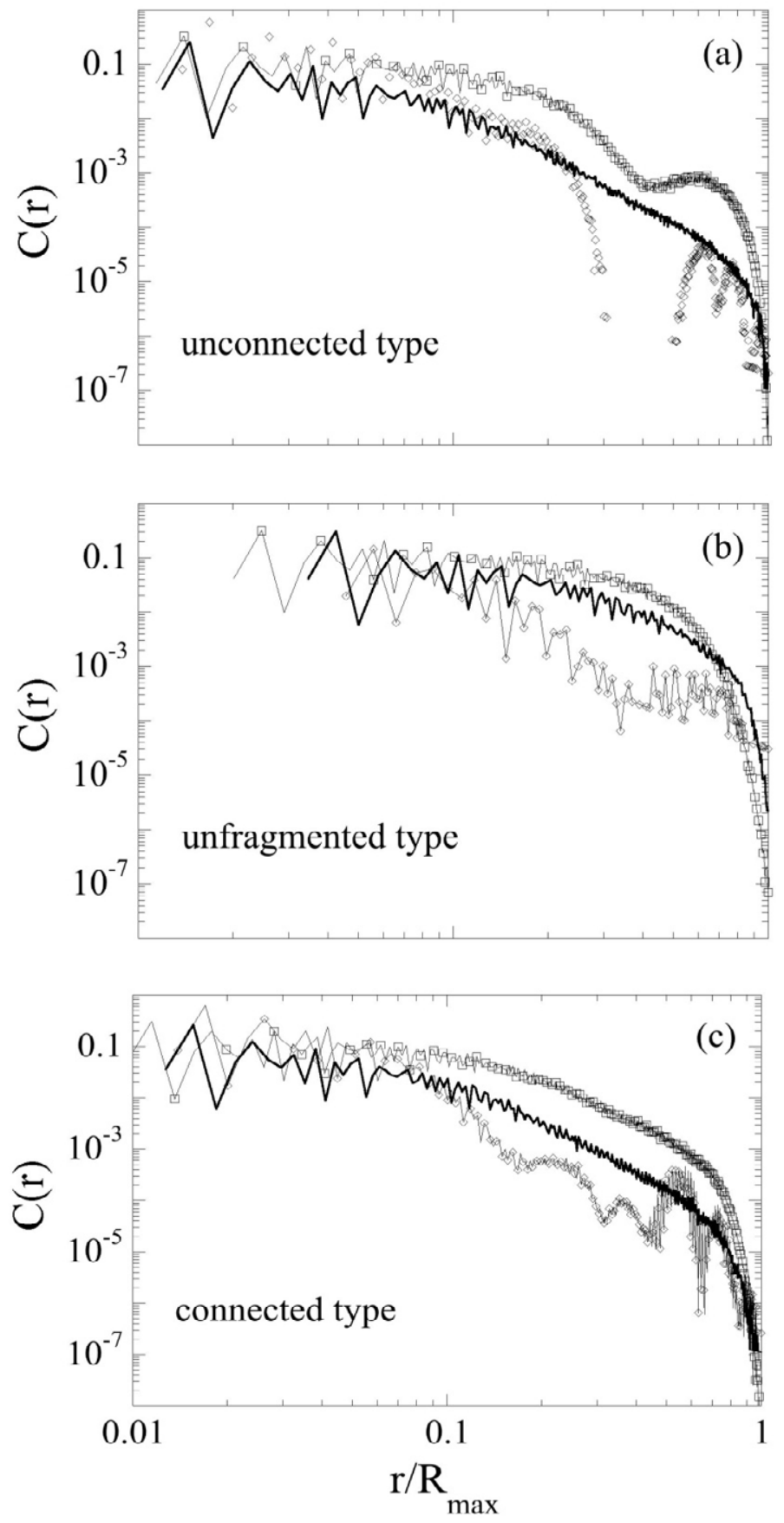

Figure 8 
(a)

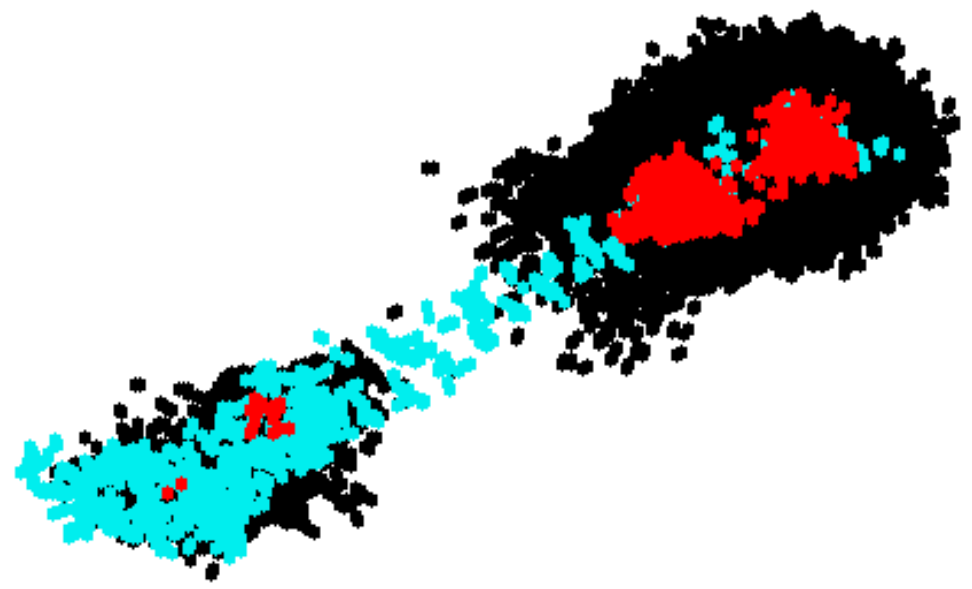

(b)

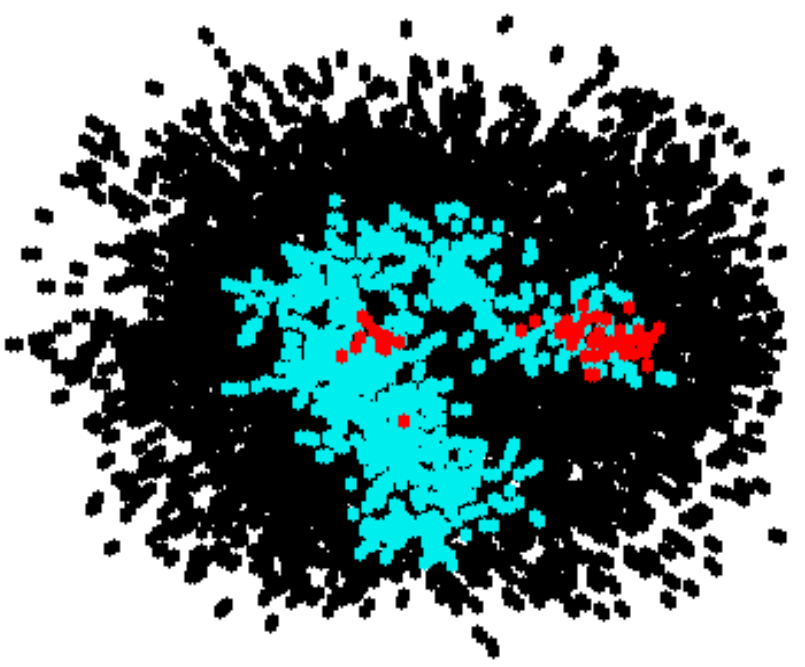

(c)

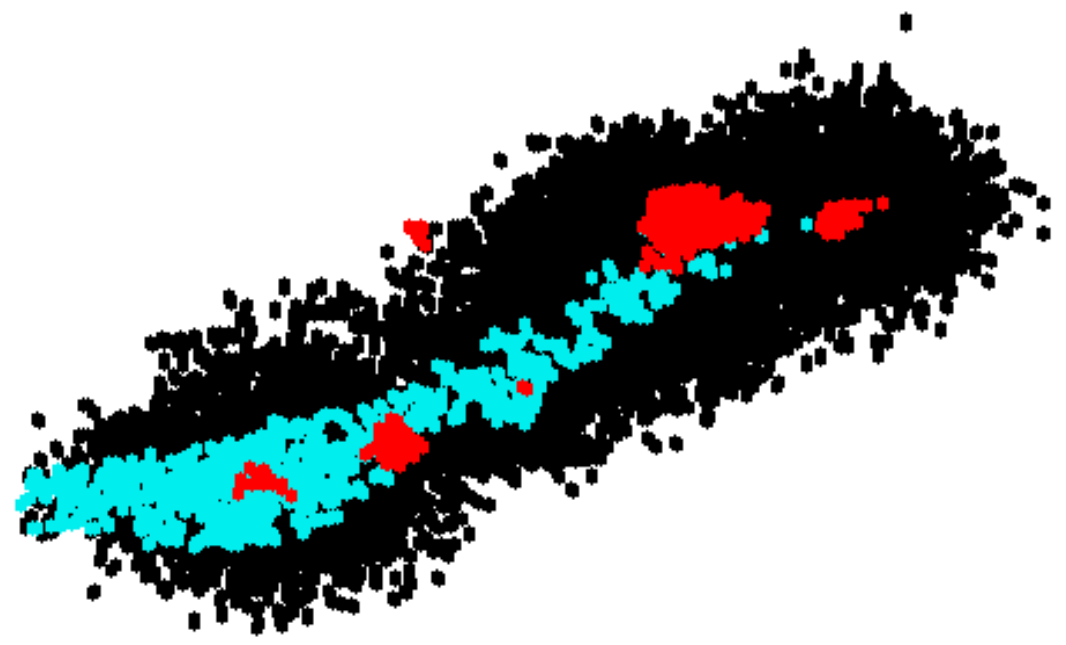

Figure 9 


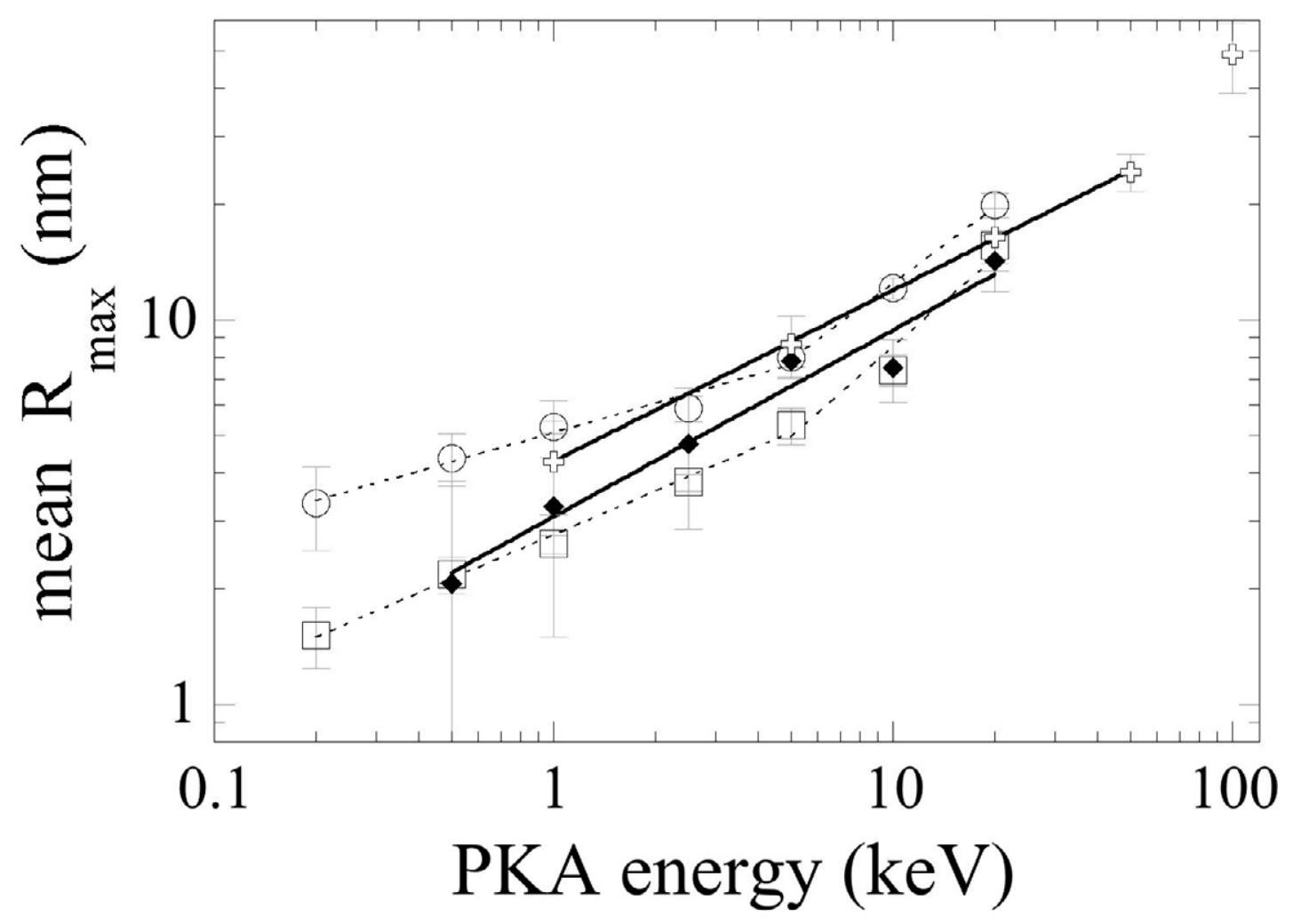

Figure 10 


\section{ACCEPTED MANUSCRIPT}

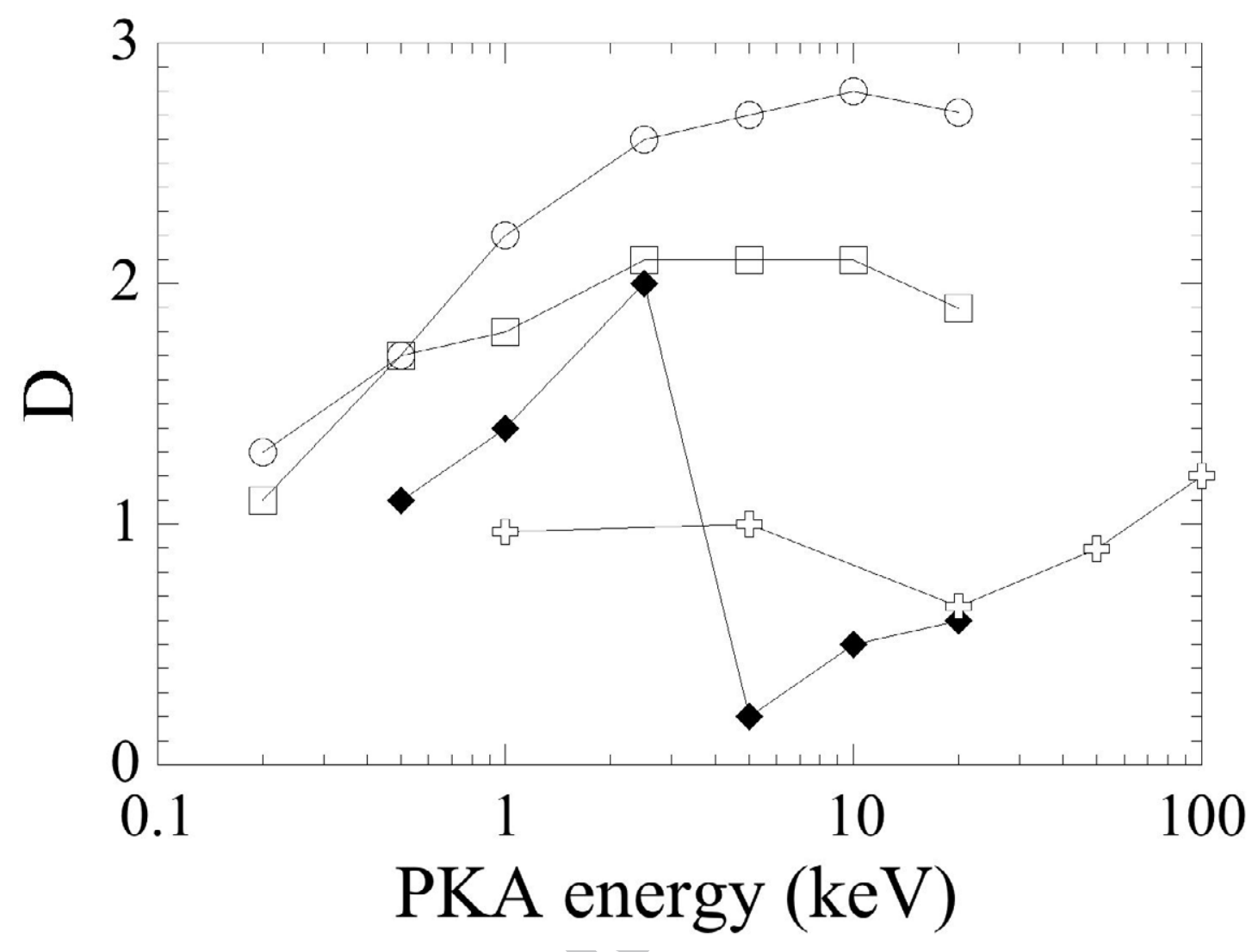

Figure 11 


\section{Highlights}

The subcascades formation in copper and iron is studied by molecular dynamics method.

A two-point density correlation function is a tool for detecting subcascade formation.

A fractal analysis served to determine the subcascade formation threshold energy.

The temporal evolution of displacement cascades is investigated. 